CERN-TH/97-221

hep-th/9709174

\title{
Finite Energy Solutions in Three-Dimensional Heterotic String Theory
}

\author{
Michèle Bourdeau and Gabriel Lopes Cardoso [ \\ Theory Division, CERN, CH-1211 Geneva 23, Switzerland
}

\begin{abstract}
We show that a large class of supersymmetric solutions to the low-energy effective field theory of heterotic string theory compactified on a seven torus can have finite energy, which we compute. The mechanism by which these solutions are turned into finite energy solutions is similar to the one occurring in the context of four-dimensional stringy cosmic string solutions. We also describe the solutions in terms of intersecting eleven-dimensional M-branes, M-waves and M-monopoles.
\end{abstract}

CERN-TH/97-221

September 1997

\footnotetext{
${ }^{1}$ Email: bourdeau@mail.cern.ch, cardoso@mail.cern.ch
} 


\section{Introduction}

A large class of supersymmetric soliton solutions in string theory have by now been constructed in various dimensions (for a review see for instance [1, 2, 3] and references therein), as these play a fundamental role in duality studies. While most of the recent work on supersymmetric solutions in string theory has been done in dimensions higher or equal to four, some heterotic supersymmetric solutions have now been determined in three space-time dimensions [4, 5]. The solutions presented in [4, 5] are static supersymmetric solutions of the low-energy effective field theory of heterotic string theory compactified on a seven-torus, which is described by a three dimensional supergravity theory with eight local supersymmetries [6, [1].

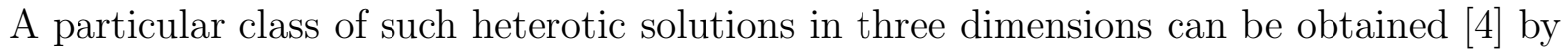
compactifying the four-dimensional string solutions of [7, 8] on a circle. In [4] it was shown that the resulting three dimensional solutions can be turned into finite energy solutions by utilizing a mechanism first discussed in the context of four-dimensional stringy cosmic string solutions [9]. It was further conjectured in [4] that this mechanism should also apply to other three-dimensional supersymmetric solutions. We will see that the same mechanism can indeed be used for turning the solutions constructed in [0] into finite energy solutions.

The construction of the supersymmetric solutions given in [5] was achieved by solving the associated Killing spinor equations in three dimensions. These Killing spinors have a priori 16 real degrees of freedom which, however, get reduced by imposing certain contraints specific to each of the solutions. Up to three such independent conditions $(m=1,2,3)$ can be imposed on the Killing spinors, resulting in Killing spinors with $1 / 2^{m}$ of 16 real degrees of freedom. The associated solutions were referred to as preserving $1 / 2^{m}$ of $N=8, D=3$ supersymmetry.

The solutions constructed in [5] are, however, only valid asymptotically, that is at large spatial distances. Thus, they should get modified in such a way as to render them well behaved at finite distances $\llbracket$. We will see that this is indeed possible by turning them into finite energy solutions.

The solutions constructed in 4 , 5 have a ten-dimensional heterotic interpretation in terms of intersections of fundamental strings, NS 5-branes, waves and Kaluza-Klein

monopoles (or, equivalently, an eleven dimensional interpretation in terms of intersections of M-branes, M-waves and M-monopoles). Let us for instance consider the solutions constructed in [5]. They fall into two classes. Namely, they either carry one or two electric 
charges. We will show below that the solutions carrying one electric charge have a tendimensional interpretation in terms of a wave and up to three orthogonally intersecting NS 5-branes. The solutions carrying two electric charges, on the other hand, have a ten-dimensional interpretation in terms of a fundamental string, a wave and up to three orthogonally intersecting NS 5-branes as well as up to three Kaluza-Klein monopoles. On the other hand, the three-dimensional solutions obtained [4] by compactifying the four-dimensional heterotic string solutions of [7, 8] on a circle have a ten-dimensional interpretation in terms of a fundamental string and up to three orthogonally intersecting NS 5-branes. We note here that the solutions constructed in [4, 5] should also have an equivalent description in terms of configurations of type IIB p-branes (such as the 7-brane of [10]) wrapped around $K 3 \times T_{3}$.

The ten dimensional space-time line element describing orthogonally intersecting strings, 5-branes, waves and Kaluza-Klein monopoles are given in terms of harmonic functions which depend on some of the overall transverse directions. These line elements, when compactified down to three dimensions, give rise to three dimensional space-time line elements which are again given in terms of the same harmonic functions. In three spacetime dimensions, a harmonic function $H(z, \bar{z})$ does not however get determined by the condition of it being harmonic. Denoting the two spatial dimensions by $z$ and $\bar{z}$, one has

$$
\partial_{z} \partial_{\bar{z}} H=0 \rightarrow H=f(z)+\bar{f}(\bar{z})
$$

where $f(z)$ is an a priori arbitrary holomorphic function. Thus, we expect that those heterotic supersymmetric three-dimensional solutions which have an M-theory description in terms of orthogonally intersecting M-branes, M-monopoles and M-waves should also be expressed in terms of holomorphic functions $f(z)$. Demanding that these supersymmetric solutions have a certain behaviour at spatial infinity $(z \rightarrow \infty)$ determines the form of $f(z)$ at large $z$. For instance, the solutions presented in [5] have an asymptotic behaviour corresponding to $f(z) \propto \ln z$. At finite distance these asymptotic solutions become illdefined and so need to be modified. The associated corrections will all be encoded in $f(z)$. Requiring the solutions to have finite energy as well as the above asymptotic behaviour will determine $f(z)$ to be given by $f(z) \propto j^{-1}(z)$. The resulting modified solutions are then well-behaved at finite distances. Asymptotically, the associated coupling constant $g^{2}=e^{2 \phi}$ is weak, whereas at finite distances it becomes strong.

This paper is organised as follows. In section 2 we review some properties of the lowenergy effective action of heterotic string theory compactified on a seven-dimensional torus [4]. In section 3 we give the Killing spinor equations associated to the threedimensional heterotic low-energy effective Lagrangian and we present some results. 
In section 4 we first review some of the static supersymmetric solutions carrying two electric charges, whose asymptotic form was given in [5]. These solutions are labelled by an integer $n$ with $n=1,2,3,4$. We then show how these solutions can be rewritten in terms of holomorphic functions $f(z)$, and check that the associated three-dimensional Killing spinor equations are solved for any arbitrary holomorphic $f(z)$. Demanding that these solutions have finite energy as well as the asymptotic behaviour that was found in [5] determines $\hat{f}(z)=\frac{n+1}{\pi} f(z)$ to be given by $j(\hat{f}(z))=z$. The dilaton is, for any $n$, determined to be

$$
e^{-\phi}=f(z)+\bar{f}(\bar{z})
$$

Next, we compute the energy $E$ carried by these solutions and find that (in units where $\left.8 \pi G_{N}=1\right) E=2 n \frac{\pi}{6}$. The solutions presented in [5] describe one-center solutions. They can be straightforwardly generalized to multi-center solutions via $j(\hat{f}(z))=P(z) / Q(z)$ [9], where $P(z)$ and $Q(z)$ are polynomials in $z$ with no common factors.

In section 5, we repeat the analysis given in section 4 for some of the static solutions now carrying one electric charge with asymptotic form given in [5]. These solutions are again labelled by an integer $n$ with $n=1,2,3,4$. As in the case of two electric charges, we show how these solutions can be rewritten in terms of holomorphic functions $f(z)$, and we check again that the associated Killing spinor equations are solved for any arbitrary holomorphic $f(z)$. As before, demanding that these solutions have finite energy as well as the asymptotic behaviour found in [5] determines $\hat{f}(z)=\frac{n+2}{2 \pi} f(z)$ to be again given by $j(\hat{f}(z))=z$. This time, however, the dilaton is, for any $n$, determined to be

$$
e^{-2 \phi}=f(z)+\bar{f}(\bar{z})
$$

We find that the energy $E$ associated with these solutions is (in units where $8 \pi G_{N}=1$ ) $E=n \frac{\pi}{6}$. This is half of the amount carried by the solutions with two electric charges. These solutions can again be straightforwardly generalized to multi-center solutions via $j(\hat{f}(z))=P(z) / Q(z)$.

In section 6, we present the eleven-dimensional interpretation in terms of orthogonally intersecting M-branes, M-waves and M-monopoles for the solutions discussed in sections 4 and 5.

Finally, in section 7, we present our conclusions.

We use the same conventions as in [5]. 


\section{The three-dimensional effective action}

The effective low-energy field theory of the ten-dimensional heterotic string compactified on a seven-dimensional torus is obtained from reducing the ten-dimensional $N=1$ supergravity theory coupled to $U(1)^{16}$ super Yang-Mills multiplets (at a generic point in the moduli space) [11, 12, 4]. The massless ten-dimensional bosonic fields are the metric $G_{M N}^{(10)}$, the antisymmetric tensor field $B_{M N}^{(10)}$, the $U(1)$ gauge fields $A_{M}^{(10) I}$ and the scalar dilaton $\Phi^{(10)}$ with $(0 \leq M, N \leq 9, \quad 1 \leq I \leq 16)$. The field strengths are $F_{M N}^{(10) I}=\partial_{M} A_{N}^{(10) I}-\partial_{N} A_{M}^{(10) I}$ and $H_{M N P}^{(10)}=\left(\partial_{M} B_{N P}^{(10)}-\frac{1}{2} A_{M}^{(10) I} F_{N P}^{(10) I}\right)+$ cyclic permutations of $M, N, P$.

The bosonic part of the ten-dimensional action is

$$
\begin{array}{r}
\mathcal{S} \propto \int d^{10} x \sqrt{-G^{(10)}} e^{-\Phi^{(10)}\left[\mathcal{R}^{(10)}+G^{(10) M N} \partial_{M} \Phi^{(10)} \partial_{N} \Phi^{(10)}\right.} \\
\left.-\frac{1}{12} H_{M N P}^{(10)} H^{(10) M N P}-\frac{1}{4} F_{M N}^{(10) I} F^{(10) I M N}\right] .
\end{array}
$$

The reduction to three dimensions [6, 12, 4] introduces the graviton $g_{\mu \nu}$, the dilaton $\phi \equiv \Phi^{(10)}-\ln \sqrt{\operatorname{det} G_{m n}}$, with $G_{m n}$ the internal 7D metric, $30 U(1)$ gauge fields $A_{\mu}^{(a)} \equiv$ $\left(A_{\mu}^{(1) m}, A_{\mu m}^{(2)}, A_{\mu}^{(3) I}\right) \quad(a=1, \ldots, 30, m=1, \ldots, 7, I=1, \ldots, 16)$, where $A_{\mu}^{(1) m}$ are

the 7 Kaluza-Klein gauge fields coming from the reduction of $G_{M N}^{(10)}, A_{\mu m}^{(2)} \equiv B_{\mu m}+$ $B_{m n} A_{\mu}^{(1) n}+\frac{1}{2} a_{m}^{I} A_{\mu}^{(3) I}$ are the 7 gauge fields coming from the reduction of $B_{M N}^{(10)}$ and $A_{\mu}^{(3) I} \equiv A_{\mu}^{I}-a_{m}^{I} A_{\mu}^{(1) m}$ are the 16 gauge fields from $A_{M}^{(10) I}$.

The field strengths $F_{\mu \nu}^{(a)}$ are given by $F_{\mu \nu}^{(a)}=\partial_{\mu} A_{\nu}^{(a)}-\partial_{\nu} A_{\mu}^{(a)}$. Finally, $B_{M N}^{(10)}$ induces the two-form field $B_{\mu \nu}$ with field strength $H_{\mu \nu \rho}=\partial_{\mu} B_{\nu \rho}-\frac{1}{2} A_{\mu}^{(a)} L_{a b} F_{\nu \rho}^{(b)}+$ cyclic permutations.

The 161 scalars $G_{m n}, a_{m}^{I}$ and $B_{m n}$ can be arranged into a $30 \times 30$ matrix $M$ (we use here the conventions of [12])

$$
M=\left(\begin{array}{ccc}
G^{-1} & -G^{-1} C & -G^{-1} a^{T} \\
-C^{T} G^{-1} & G+C^{T} G^{-1} C+a^{T} a & C^{T} G^{-1} a^{T}+a^{T} \\
-a G^{-1} & a G^{-1} C+a & I_{16}+a G^{-1} a^{T}
\end{array}\right)
$$

where $G \equiv\left[G_{m n}\right], C \equiv\left[\frac{1}{2} a_{m}^{I} a_{n}^{I}+B_{m n}\right]$ and $a \equiv\left[a_{m}^{I}\right]$.

We have $M L M^{T}=L, \quad M^{T}=M, \quad L^{-1}=L$, where

$$
L=\left(\begin{array}{ccc}
0 & I_{7} & 0 \\
I_{7} & 0 & 0 \\
0 & 0 & I_{16}
\end{array}\right)
$$


We use the following ansatz for the Kaluza-Klein $10 \mathrm{D}$ vielbein $E_{M}^{A}$ and inverse vielbein $E_{A}^{M}$, in the string frame

$$
E_{M}^{A}=\left(\begin{array}{cl}
e^{\phi} e_{\mu}^{\alpha} & A_{\mu}^{(1) m} e_{m}^{a} \\
0 & e_{m}^{a}
\end{array}\right), \quad E_{A}^{M}=\left(\begin{array}{cc}
e^{-\phi} e_{\alpha}^{\mu} & -e^{-\phi} e_{\alpha}^{\mu} A_{\mu}^{(1) m} \\
0 & e_{a}^{m}
\end{array}\right),
$$

where $e_{m}^{a}$ is the internal and $e_{\mu}^{\alpha}$ the space-time vielbein in the Einstein frame (the relation between string metric $G_{\mu \nu}$ and Einstein metric $g_{\mu \nu}$ in three dimensions is $G_{\mu \nu}=e^{2 \phi} g_{\mu \nu}$ ).

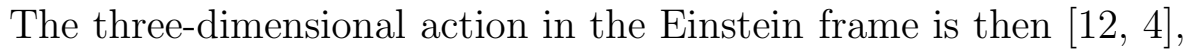

$$
\begin{aligned}
\mathcal{S}= & \frac{1}{4} \int d^{3} x \sqrt{-g}\left\{\mathcal{R}-g^{\mu \nu} \partial_{\mu} \phi \partial_{\nu} \phi-\frac{1}{12} e^{-4 \phi} g^{\mu \mu^{\prime}} g^{\nu \nu^{\prime}} g^{\rho \rho^{\prime}} H_{\mu \nu \rho} H_{\mu^{\prime} \nu^{\prime} \rho^{\prime}}\right. \\
& \left.-\frac{1}{4} e^{-2 \phi} g^{\mu \mu^{\prime}} g^{\nu \nu^{\prime}} F_{\mu \nu}^{(a)}(L M L)_{a b} F_{\mu^{\prime} \nu^{\prime}}^{(b)}+\frac{1}{8} g^{\mu \nu} \operatorname{Tr}\left(\partial_{\mu} M L \partial_{\nu} M L\right)\right\},
\end{aligned}
$$

where $a=1, \ldots, 30$.

This action is invariant under the $O(7,23)$ transformations

$$
M \rightarrow \tilde{\Omega} M \tilde{\Omega}^{T}, \quad A_{\mu}^{(a)} \rightarrow \tilde{\Omega}_{a b} A_{\mu}^{(b)}, \quad g_{\mu \nu} \rightarrow g_{\mu \nu}, \quad B_{\mu \nu} \rightarrow B_{\mu \nu}, \quad \phi \rightarrow \phi, \quad \tilde{\Omega}^{T} L \tilde{\Omega}=L
$$

where $\tilde{\Omega}$ is a $30 \times 30 O(7,23)$ matrix.

The equations of motion for $A_{\mu}^{(a)}, \phi, H^{\mu \nu \rho}$ and $g^{\mu \nu}$ are, respectively,

$$
\begin{aligned}
& \partial_{\mu}\left(e^{-2 \phi} \sqrt{-g}(L M L)_{a b} F^{(b) \mu \nu}\right)+\frac{1}{2} e^{-4 \phi} \sqrt{-g} L_{a b} F_{\mu \rho}^{(b)} H^{\nu \mu \rho}=0 \\
& D_{\mu} D^{\mu} \phi+\frac{1}{4} e^{-2 \phi} F_{\mu \nu}^{(a)}(L M L)_{a b} F^{\mu \nu(b)}+\frac{1}{6} e^{-4 \phi} H^{\mu \nu \rho} H_{\mu \nu \rho}=0 \\
& \partial_{\mu}\left(\sqrt{-g} e^{-4 \phi} H^{\mu \nu \rho}\right)=0 \\
& \mathcal{R}_{\mu \nu}=\partial_{\mu} \phi \partial_{\nu} \phi+\frac{1}{2} e^{-2 \phi} F_{\mu \rho}^{(a)}(L M L)_{a b} F_{\nu}^{\rho(b)}-\frac{1}{8} \operatorname{Tr}\left(\partial_{\mu} M L \partial_{\nu} M L\right) \\
& \quad-\frac{1}{4} e^{-2 \phi} g_{\mu \nu} F_{\rho \tau}^{(a)}(L M L)_{a b} F^{\rho \tau(b)}+\frac{1}{4} e^{-4 \phi} H_{\mu}^{\tau \sigma} H_{\nu \tau \sigma}-\frac{1}{6} g_{\mu \nu} e^{-4 \phi} H^{\tau \sigma \rho} H_{\tau \sigma \rho} .
\end{aligned}
$$

We will, in the following, consider backgrounds where $H_{\mu \nu \rho}=0$.

Consider a static solution with space-time line element of the form

$$
d s^{2}=-d t^{2}+g_{x x}\left(d x^{2}+d y^{2}\right)=-d t^{2}+2 g_{z \bar{z}} d z d \bar{z}
$$

where $z=x+i y$. Its energy $E$ can be computed from (2.7)-(2.10) and is given by

$$
E=\frac{1}{8 \pi G_{N}} \int d^{2} x g_{x x} T_{t t}=\frac{i}{8 \pi G_{N}} \int d z d \bar{z} g_{z \bar{z}} T_{t t}=\frac{i}{16 \pi G_{N}} \int d z d \bar{z} g_{z \bar{z}} \mathcal{R}
$$

where $8 \pi G_{N} T_{\mu \nu}=\mathcal{R}_{\mu \nu}-\frac{1}{2} g_{\mu \nu} \mathcal{R}$. Here $G_{N}$ denotes the three-dimensional gravitational constant. We will in the following set $8 \pi G_{N}=1$. 
From the equations of motion for the gauge fields $A_{\mu}^{(a)}(2.7)$ one can define a set of scalar fields $\Psi^{a}, a=1, \ldots, 30$, through [4]

$$
\begin{aligned}
& \sqrt{-g} e^{-2 \phi} g^{\mu \mu^{\prime}} g^{\nu \nu^{\prime}}(M L)_{a b} F_{\mu^{\prime} \nu^{\prime}}^{(b)}=\epsilon^{\mu \nu \rho} \partial_{\rho} \Psi^{a}, \\
& F^{(a) \mu \nu}=\frac{1}{\sqrt{-g}} e^{2 \phi}(M L)_{a b} \epsilon^{\mu \nu \rho} \partial_{\rho} \Psi^{b} .
\end{aligned}
$$

Then, from the Bianchi identity $\epsilon^{\mu \nu \rho} \partial_{\mu} F_{\nu \rho}^{(a)}=0$,

$$
D^{\mu}\left(e^{2 \phi}(M L)_{a b} \partial_{\mu} \Psi^{b}\right)=0 .
$$

Following [4], the charge quantum numbers of elementary string excitations are characterized by a 30-dimensional vector $\vec{\alpha} \in \Lambda_{30}$. The asymptotic value of the field strength $F_{\mu \nu}^{(a)}$ associated with such an elementary particle can be calculated to be [4]

$$
\sqrt{-g} F^{(a) t r} \simeq-\frac{1}{2 \pi} e^{2 \phi} M_{a b} \alpha^{b}
$$

The asymptotic form of $\Psi^{a}$ is then

$$
\Psi^{a} \simeq-\frac{\theta}{2 \pi} L_{a b} \alpha^{b}+\text { constant }
$$

It can be shown [4 that the matrix $M$, the $\Psi$ 's and the dilaton can be assembled into a matrix $\mathcal{M}$ describing the coset $\frac{O(8,24)}{O(8) \times O(24)}$. The low energy effective three-dimensional field theory is then actually invariant under $O(8,24)$ transformations. An $O(8,24 ; \mathbb{Z})$ subgroup of this group is a symmetry of the full string theory [四].

\section{The Killing spinor equations}

In ten dimensions, the supersymmetry transformation rules for the gaugini $\chi^{I}$, dilatino $\lambda$ and gravitino $\psi_{M}$ are, in the string frame, given by [13, 14, 15, 16, 17]

$$
\begin{aligned}
& \delta \chi^{I}=\frac{1}{2} F_{M N}^{I} \Gamma^{M N} \varepsilon, \\
& \delta \lambda=-\frac{1}{2} \Gamma^{M} \partial_{M} \Phi \varepsilon+\frac{1}{12} H_{M N P} \Gamma^{M N P} \varepsilon, \\
& \delta \psi_{M}=\partial_{M} \varepsilon+\frac{1}{4}\left(\omega_{M A B}-\frac{1}{2} H_{M A B}\right) \Gamma^{A B} \varepsilon .
\end{aligned}
$$

These equations were reduced to three dimensions in the Einstein frame in [5]. In the following, we restrict ourselves to backgrounds with $H_{\mu \nu \rho}=0$ and $a_{m}^{I}=0$. The associated three-dimensional Killing spinor equations become

$$
\delta \chi^{I}=\frac{1}{2} e^{-2 \phi} F_{\mu \nu}^{(3) I} \gamma^{\mu \nu} \varepsilon
$$




$$
\begin{aligned}
\delta \lambda= & -\frac{1}{2} e^{-\phi} \partial_{\mu}\left\{\phi+\ln \operatorname{det} e_{m}^{a}\right\} \gamma^{\mu} \otimes \mathbf{I}_{8} \varepsilon+\frac{1}{4} e^{-2 \phi}\left[-B_{m n} F_{\mu \nu}^{(1) n}+F_{\mu \nu m}^{(2)}\right] \gamma^{\mu \nu} \gamma^{4} \otimes \Sigma^{m} \varepsilon \\
& +\frac{1}{4} e^{-\phi} \partial_{\mu} B_{m n} \gamma^{\mu} \otimes \Sigma^{m n} \varepsilon, \\
\delta \psi_{\mu}= & \partial_{\mu} \varepsilon+\frac{1}{4} \omega_{\mu \alpha \beta} \gamma^{\alpha \beta} \varepsilon+\frac{1}{4}\left(e_{\mu \alpha} e_{\beta}^{\nu}-e_{\mu \beta} e_{\alpha}^{\nu}\right) \partial_{\nu} \phi \gamma^{\alpha \beta} \varepsilon+\frac{1}{8}\left(e_{a}^{n} \partial_{\mu} e_{n b}-e_{b}^{n} \partial_{\mu} e_{n a}\right) \mathbf{I}_{4} \otimes \Sigma^{a b} \varepsilon \\
& -\frac{1}{4} e^{-\phi}\left[e_{a}^{m} F_{\mu \nu(m)}^{(2)}-e_{m a} F_{\mu \nu}^{(1) m}\right] \gamma^{\nu} \gamma^{4} \otimes \Sigma^{a} \varepsilon-\frac{1}{8} \partial_{\mu} B_{m n} \mathbf{I}_{4} \otimes \Sigma^{m n} \varepsilon \\
& +\frac{1}{4} e^{-\phi} B_{m n} F_{\mu \nu}^{(1) n} \gamma^{\nu} \gamma^{4} \otimes \Sigma^{m} \varepsilon, \\
\delta \psi_{d}= & -\frac{1}{4} e^{-\phi}\left(e_{d}^{m} \partial_{\mu} e_{m a}+e_{a}^{m} \partial_{\mu} e_{m d}\right) \gamma^{\mu} \gamma^{4} \otimes \Sigma^{a} \varepsilon+\frac{1}{8} e^{-2 \phi} e_{d}^{m} B_{m n} F_{\mu \nu}^{(1) n} \gamma^{\mu \nu} \varepsilon \\
& +\frac{1}{4} e^{-\phi} e_{d}^{m} e_{a}^{n} \partial_{\mu} B_{m n} \gamma^{\mu} \gamma^{4} \otimes \Sigma^{a} \varepsilon-\frac{1}{8} e^{-2 \phi}\left[e_{m d} F_{\mu \nu}^{(1) m}+e_{d}^{m} F_{\mu \nu m}^{(2)}\right] \gamma^{\mu \nu} \varepsilon
\end{aligned}
$$

where $\delta \psi_{d} \equiv e_{d}^{m} \delta \psi_{m}$ denotes the variation of the internal gravitini.

Static solutions to the Killing spinor equations can be constructed by setting the supersymmetry variations of the fermionic fields to zero. This ensures that the bosonic configurations so obtained are supersymmetric.

The supersymmetric static solutions we will be discussing in the following sections will either carry one or two electric charges. They have the following space-time line elements:

$$
d s^{2}=-d t^{2}+H^{c n}(\omega, \bar{\omega}) d \omega d \bar{\omega} \quad, \quad c=1,2 \quad, \quad n=1,2,3,4
$$

where $\omega=\hat{r}+i \hat{\theta}$, and where the solutions carrying one (two) electric charge have $c=1$ $(c=2)$. $H(\omega, \bar{\omega})$ denotes a harmonic function:

$$
\partial_{\omega} \partial_{\bar{\omega}} H=0 \longrightarrow H(\omega, \bar{\omega})=f(\omega)+f(\bar{\omega})
$$

The dilaton is found to be

$$
e^{-2 \phi}=H^{c}
$$

In all cases, we will make the following ansatz for the Killing spinors

$$
\varepsilon=\epsilon \otimes \chi
$$

where $\epsilon^{T}=\left(\epsilon_{1}, \epsilon_{2}, \epsilon_{3}, \epsilon_{4}\right)$ is a $S O(1,2)$ spinor and $\chi$ is a $S O(7)$ spinor of the internal space. We will be able to solve the Killing spinor equations by imposing the following two conditions on $\epsilon$ [5]:

$$
\gamma^{1} \epsilon=i p \gamma^{2} \epsilon, \quad \gamma^{1} \epsilon=\tilde{p} J \gamma^{2} \gamma^{4} \epsilon
$$


where $p= \pm, \tilde{p}= \pm$. Here we used that $\gamma^{\mu}=\gamma^{\alpha} e_{\alpha}^{\mu}, \alpha=0,1,2$.

It follows that

$$
\epsilon=\tilde{\epsilon}(\omega, \bar{\omega})\left(\begin{array}{c}
i p \\
1 \\
\tilde{p} \\
-i p \tilde{p}
\end{array}\right)
$$

and, hence, $\epsilon$ contains only two real independent degrees of freedom. $\chi$, on the other hand, contains eight real degrees of freedom; thus there are a priori a total of 16 real degrees of freedom. These will be further reduced by conditions on $\chi$ specific to each case considered. Up to three such independent conditions $(m=1,2,3)$ can be imposed on $\chi$, thereby allowing for the construction of solutions whose Killing spinors have $1 / 2^{m}$ of 16 real degrees of freedom. The solutions with $n=1$ and $n=2$ have Killing spinors with 8 and 4 real degrees of freedom, respectively. The solutions with $n=3$ and $n=4$ both have Killing spinors with 2 real degrees of freedom.

In all cases considered in the following, we find that

$$
\tilde{\epsilon}=e^{\frac{\phi}{2}}=H^{-\frac{c}{4}}
$$

up to a multiplicative constant.

\section{Supersymmetric solutions carrying two electric charges}

Here, we will consider the class of solutions carrying two electric charges presented in [5]. The solutions given there are well behaved only at large spatial distances, and hence need to be modified at finite distances.

The solutions in this class are labelled by an integer $n(n=1,2,3,4)$. The associated dilaton was given by (in a specific coordinate system) [5]

$$
e^{\phi}=\frac{n+1}{a \ln r}
$$

whereas the associated space-time line element was

$$
d s^{2}=-d t^{2}+\frac{a^{2}}{r^{2}}\left(\frac{a \ln r}{n+1}\right)^{2 n}\left(d r^{2}+r^{2} d \theta^{2}\right)
$$

Here, $a=\frac{n+1}{2 \pi} \sqrt{\left|\alpha_{i} \alpha_{i+7}\right|}$, where $\alpha_{i}$ and $\alpha_{i+7}$ denote the two electric charges. The ith component of the internal metric $G_{m n}$ was given by $G_{i i}=\left|\frac{\alpha_{i}}{\alpha_{i+7}}\right|$. The gauge fields 
strengths, or equivalently $\Psi^{i}$ and $\Psi^{i+7}$, were taken to have the asymptotic form given in (2.16). Introducing complex coordinates, $\omega=a \ln z=a(\ln r+i \theta)$, then yields

$$
d s^{2}=-d t^{2}+H^{2 n} d \omega d \bar{\omega} \quad, \quad e^{-\phi}=H \quad,
$$

where $H=\frac{a \ln r}{n+1}=\frac{\omega+\bar{\omega}}{2(n+1)}$. Inspection of (4.1) shows that the solution becomes ill defined as $r \rightarrow 1$ and thus it should get modified at finite distances. The asymptotic form of $H$ suggests the replacement

$$
H=\frac{\omega+\bar{\omega}}{2(n+1)} \longrightarrow H=f(\omega)+\bar{f}(\bar{\omega})
$$

Similarly, the asymptotic form of the $\Psi^{i}$ given in (2.16) suggests the replacement

$$
\begin{aligned}
\Psi^{i} & =\frac{\alpha_{i+7}}{4 \pi a} i(\omega-\bar{\omega}) \longrightarrow \Psi^{i}=\frac{(n+1) \alpha_{i+7}}{2 \pi a} i(f-\bar{f}), \\
\Psi^{i+7} & =\frac{\alpha_{i}}{4 \pi a} i(\omega-\bar{\omega}) \longrightarrow \Psi^{i+7}=\frac{(n+1) \alpha_{i}}{2 \pi a} i(f-\bar{f}) .
\end{aligned}
$$

Denoting the imaginary part of $f$ by $\hat{\Psi}, \hat{\Psi}=-i(f-\bar{f})$, we have

$$
f=\frac{1}{2}\left(e^{-\phi}+i \hat{\Psi}\right)
$$

Using $G_{i i}=\left|\frac{\alpha_{i}}{\alpha_{i+7}}\right|$, we can rewrite $\Psi^{i}$ and $\Psi^{i+7}$ in the following way

$$
\Psi^{i}=-\eta_{\alpha_{i+7}} \sqrt{G^{i i}} \hat{\Psi} \quad, \quad \Psi^{i+7}=-\eta_{\alpha_{i}} \sqrt{G_{i i}} \hat{\Psi}
$$

where $\eta_{\alpha_{i+7}}=-\eta_{\alpha_{i}}$ denotes the sign of the two charges $\alpha_{i}$ and $\alpha_{i+7}$. Thus we see that the $\Psi$ 's can be reexpressed in terms of the internal metric and of $\hat{\Psi}$.

Using that $\partial_{\bar{\omega}} f=0$ it can be shown that the associated field strengths are given by

$$
F_{t \beta}^{(1) i}=\eta_{\alpha_{i}} \sqrt{G^{i i}} \frac{\partial_{\beta} H}{H^{2}} \quad, \quad F_{t \beta i}^{(2)}=-\eta_{\alpha_{i}} \sqrt{G_{i i}} \frac{\partial_{\beta} H}{H^{2}} \quad, \quad \beta=\hat{r}, \hat{\theta},
$$

where $\omega=a(\ln r+i \theta)=\hat{r}+i \hat{\theta}$.

We now discuss the solutions in more detail. Let us first consider the case where $n=1$. The associated Killing spinor has 1/2 of 16 real degrees of freedom. The solution is characterised by the fact that the internal vielbein $e_{m}^{a}$ is diagonal and constant and that $B_{m n}=0$ [5]. The solution presented in [5], which has line element (4.2), should be modified so as to render the solution well behaved at finite distances. This modification is given by (4.3), (4.4) and (4.5). It can then be checked that the Killing spinor equations (3.2) are satisfied for any arbitrary holomorphic $f$. The Killing spinor is given by (3.6) and (3.8) subject to one additional condition on $\chi$ given in [5]. Thus, the requirement of supersymmetry alone does not determine $f$. 
Next, consider the case where $n=2$. The associated Killing spinor has $1 / 4$ of 16 real degrees of freedom. The solution is characterised by the fact that now there is one nonconstant off-diagonal entry in the internal metric as well as one non-constant entry in the $B_{m n}$-matrix. As an example, consider the case where the two electric charges are taken to be $\alpha_{4}$ and $\alpha_{11}$, and where the background fields $G_{m n}$ and $B_{m n}$ are given by [5]

$$
\begin{aligned}
& \left(\begin{array}{cccccc}
G^{11} & G^{12} & 0 & 0 & \cdots & 0 \\
G^{21} & G^{22} & 0 & 0 & \cdots & 0 \\
0 & 0 & G^{33} & 0 & \cdots & \\
0 & 0 & 0 & G^{44} & 0 & \vdots \\
\vdots & & & & \ddots & \\
0 & & \ldots & & 0 & G^{77}
\end{array}\right)=\left(\begin{array}{cccccc}
g^{2} & -g^{2} \Upsilon_{2} & 0 & 0 & \cdots & 0 \\
-g^{2} \Upsilon_{2} & \left|\frac{\alpha_{9}}{\alpha_{2}}\right|+g^{2} \Upsilon_{2}^{2} & 0 & 0 & \cdots & 0 \\
0 & 0 & G^{33} & 0 & \cdots & \\
0 & 0 & 0 & G^{44} & 0 & \vdots \\
\vdots & & & & \ddots & \\
0 & & \ldots & & 0 & G^{77}
\end{array}\right), \\
& B=\left(B_{m n}\right)=\left(\begin{array}{ccccc}
0 & B_{12} & 0 & \cdots & 0 \\
B_{21} & 0 & & & \\
0 & & 0 & & \vdots \\
\vdots & & & \ddots & \\
0 & & \cdots & & 0
\end{array}\right)=\left(\begin{array}{ccccc}
0 & \Upsilon_{9} & 0 & \cdots & 0 \\
-\Upsilon_{9} & 0 & & & \\
0 & & 0 & & \vdots \\
\vdots & & & \ddots & \\
0 & & \cdots & & 0
\end{array}\right) \text {, }
\end{aligned}
$$

where $G^{44}=\left|\frac{\alpha_{11}}{\alpha_{4}}\right|$. The solution presented in [5] is valid only at large distances, and again it should be modified at finite distances. These modifications are given by (4.3), (4.4) and (4.5) as well as by

$$
g=\frac{D}{H} \quad, \quad \Upsilon_{2}=\frac{(n+1) \alpha_{9}}{2 \pi a} i(f-\bar{f}) \quad, \quad \Upsilon_{9}=\frac{(n+1) \alpha_{2}}{2 \pi a} i(f-\bar{f}),
$$

where $D=\frac{\sqrt{\left|\alpha_{4} \alpha_{11}\right|}}{\sqrt{\left|\alpha_{2} \alpha_{9}\right|}}$. It can again be checked that this modified background satisfies the Killing spinor equations (3.2) for any arbitrary holomorphic $f$. The associated Killing spinor is given by (3.6) and (3.8) subject to two additional constraints on $\chi$ given in [5].

Finally, consider the cases where $n=3$ and $n=4$. The associated Killing spinors both have $1 / 8$ of 16 real degrees of freedom. The solutions are characterised by the addition of one (two) additional off-diagonal entries in the internal metric $G_{m n}$ and in $B_{m n}$ for $n=3$ $(n=4)[5]$. It is straightforward to modify these solutions along similar lines as the ones discussed above, and again it can be checked that these modified solutions satisfy the Killing spinor equations (3.2).

We thus see that, in any of the above modified solutions, the modifications are all encoded in one single holomorphic function $f$.

Next, we would like to determine the holomorphic function $f$ by demanding that the modified solution have finite energy [4]. This will also render the solutions well behaved at finite distances. 
Let us first compute the energy carried by the modified solutions discussed above. The integral (2.12) is computed to be (in units where $8 \pi G_{N}=1$ )

$$
E=i 2 n \int d \omega d \bar{\omega} \frac{\partial_{\omega} f \partial_{\bar{\omega}} \bar{f}}{(f+\bar{f})^{2}}=i 2 n \int d z d \bar{z} \frac{\partial_{z} \hat{f} \partial_{\bar{z}} \overline{\hat{f}}}{(\hat{f}+\overline{\hat{f}})^{2}}
$$

where $\omega=a \ln z$ and where we have introduced $\hat{f}=\frac{n+1}{\pi} f$ for later convenience.

There is an elegant mechanism [9] for rendering the integral (4.11) finite. Let us take $z$ to be the coordinate of a complex plane. Then there is a one-to-one map from a certain domain $\mathrm{F}$ on the $\hat{f}$-plane (the so called 'fundamental' domain) to the $z$-plane. This map is known as the j-function, $j(\hat{f})=z$. By means of this map, the integral (4.11) can be pulled back from the $z$-plane to the domain $\mathrm{F}$ (the $z$-plane covers $\mathrm{F}$ exactly once). Then, by using integration by parts, this integral can be related to a line integral over the boundary of $\mathrm{F}$, which is evaluated to be 9

$$
E=2 n \frac{2 \pi}{12}=2 n \frac{\pi}{6}
$$

and, hence, is finite.

By expanding $j(\hat{f})=e^{2 \pi \hat{f}}+744+\mathcal{O}\left(e^{-2 \pi \hat{f}}\right)=z$ we see that $f(\omega)=$ $\frac{1}{2(n+1)}\left(\omega-744 e^{-\omega}+\ldots\right)$, which indeed reproduces the correct asymptotic behaviour at $\omega \rightarrow \infty$. Thus we see that, by demanding the asymptotic behaviour of $f$ to be modified to $f=\frac{\pi}{n+1} j^{-1}(z)$, the associated energy becomes finite.

We note that the solutions discussed above represent one-center solutions. They can be generalised to multi-center solutions via $j(\hat{f}(z))=P(z) / Q(z)$, where $P(z)$ and $Q(z)$ are polynomials in $z$ with no common factors. These are the analogue of the multi-string configurations discussed in [9].

It can be checked that the curvature scalar $\mathcal{R} \propto \partial_{\omega} f \partial_{\bar{\omega}} \bar{f}$ blows up at the special point $\hat{f}=1$ (at this point, the $j$-function and its derivatives are given by $j=1728, j^{\prime}=0$ ), whereas it is well behaved at the point $\hat{f}=e^{i \pi / 6}$ (at this point, the $j$-function and its derivatives are given by $j=j^{\prime}=j^{\prime \prime}=0$ ).

\section{Supersymmetric solutions carrying one electric charge}

Here we will first review the supersymmetric solutions constructed in [5] carrying one electric charge. These solutions need again to be modified at finite distances.

The solutions in this class are also labelled by an integer $n(n=1,2,3,4)$. Here the 
dilaton was

$$
e^{2 \phi}=\frac{n+2}{2 a \ln r}
$$

whereas the associated space-time line element was

$$
d s^{2}=-d t^{2}+\frac{a^{2}}{r^{2}}\left(\frac{2 a \ln r}{n+2}\right)^{n}\left(d r^{2}+r^{2} d \theta^{2}\right)
$$

Here, $a=\frac{n+2}{4 \pi}\left|\alpha_{i}\right|$, where $\alpha_{i}$ denotes the electric charge carried by the solutions. The i-th component of the internal metric $G_{m n}$ was given by $G_{i i}=\frac{2 a \ln r}{n+2}$. The gauge fields strength, or equivalently $\Psi^{i+7}$, was taken to have the asymptotic form given in (2.16). Introducing complex coordinates, $\omega=a \ln z=a(\ln r+i \theta)$, then yields

$$
d s^{2}=-d t^{2}+H^{n} d \omega d \bar{\omega} \quad, \quad e^{-2 \phi}=H \quad, \quad G_{i i}=H \quad,
$$

where $H=\frac{2 a \ln r}{n+2}=\frac{\omega+\bar{\omega}}{n+2}$. The solution again becomes ill defined as $r \rightarrow 1$ and thus needs to get modified at finite distances.

The asymptotic form of $H$ suggests the replacement

$$
H=\frac{\omega+\bar{\omega}}{n+2} \longrightarrow H=f(\omega)+\bar{f}(\bar{\omega})
$$

Similarly, the asymptotic form of $\Psi^{i+7}$ given in (2.16) suggests the replacement

$$
\Psi^{i+7}=\frac{\alpha_{i}}{4 \pi a} i(\omega-\bar{\omega}) \longrightarrow \Psi^{i+7}=\frac{(n+2) \alpha_{i}}{4 \pi a} i(f-\bar{f})=\eta_{\alpha_{i}} i(f-\bar{f})
$$

where $\eta_{\alpha_{i}}$ denotes the sign of the charge $\alpha_{i}$. Denoting the imaginary part of $f$ by $\hat{\Psi}$, $\hat{\Psi}=-i(f-\bar{f})$, yields

$$
f=\frac{1}{2}\left(e^{-2 \phi}+i \hat{\Psi}\right)
$$

as well as

$$
\Psi^{i+7}=-\eta_{\alpha_{i}} \hat{\Psi}
$$

The associated field strength is given by

$$
F_{t \beta}^{(1) i}=\eta_{\alpha_{i}} \frac{\partial_{\beta} H}{H^{2}} \quad, \quad \beta=\hat{r}, \hat{\theta}
$$

where $\omega=a(\ln r+i \theta)=\hat{r}+i \hat{\theta}$.

We will now discuss the solutions in more detail. Let us first consider the case where $n=1$. The associated Killing spinor has $1 / 2$ of 16 real degrees of freedom. The solution is characterised by the fact that the internal vielbein $e_{m}^{a}$ is diagonal and that $B_{m n}=0$ [5]. 
The solution presented in [5], which has line element (5.2), should be modified to render the solution well behaved at finite distances. This modification is given by (5.3), (5.4) and (5.5). It can then be checked that the Killing spinor equations (3.2) are satisfied for any arbitrary holomorphic $f$. The Killing spinor is given by (3.6) and (3.8) with $p=-1$, subject to one additional condition on $\chi$ given in [5]. Thus, the requirement of supersymmetry alone does not determine $f$.

Next, consider the case where $n=2$. The associated Killing spinor has $1 / 4$ of 16 real degrees of freedom. The solution is characterised by the fact that now there is one nonconstant entry in the $B_{m n}$-matrix. The internal metric $G_{m n}$ stays diagonal, however. As an example, consider the case where the electric charge is taken to be $\alpha_{4}$, and where the background fields $G_{m n}$ and $B_{m n}$ are given by [0] ]

$$
\begin{aligned}
G^{-1} & =\left(\begin{array}{cccccc}
G^{11} & 0 & 0 & 0 & \cdots & 0 \\
0 & G^{22} & 0 & 0 & \cdots & 0 \\
0 & 0 & G^{33} & 0 & \cdots & \\
0 & 0 & 0 & G^{44} & 0 & \vdots \\
\vdots & & & & \ddots & \\
0 & & \cdots & & 0 & G^{77}
\end{array}\right)=\left(\begin{array}{cccccc}
g_{1}^{2} & 0 & 0 & 0 & \cdots & 0 \\
0 & g_{2}^{2} & 0 & 0 & \cdots & 0 \\
0 & 0 & G^{33} & 0 & \cdots & \\
0 & 0 & 0 & \frac{1}{H} & 0 & \vdots \\
\vdots & & & & \ddots & \\
0 & & \cdots & & 0 & G^{77}
\end{array}\right), \\
B & =\left(\begin{array}{ccccc}
0 & B_{12} & 0 & \cdots & 0 \\
B_{21} & 0 & & & \\
0 & & 0 & & \vdots \\
\vdots & & & \ddots & 0 \\
0 & & \cdots & & 0
\end{array}\right)=\left(\begin{array}{ccccc}
0 & \Upsilon_{9} & 0 & \cdots & 0 \\
-\Upsilon_{9} & 0 & & & \\
0 & & 0 & & \vdots \\
\vdots & & & \ddots & \\
0 & & \cdots & & 0
\end{array}\right) .
\end{aligned}
$$

The solution presented in [5] is valid only at large distances, and it should be modified at finite distances. These modifications are given by (5.3), (5.4) and (5.5) as well as by

$$
g_{1}^{2}=\frac{D_{1}^{2}}{H} \quad, \quad g_{2}^{2}=\frac{D_{2}^{2}}{H} \quad, \quad \Upsilon_{9}=\frac{(n+2) \alpha_{2}}{4 \pi a} i(f-\bar{f}),
$$

where $D_{1} D_{2}=\frac{4 \pi a}{(n+2)\left|\alpha_{2}\right|}$. It can again be checked that this modified background satisfies the Killing spinor equations (3.2) for any arbitrary holomorphic $f$. The associated Killing spinor is given by (3.6) and (3.8) with $p=-1$, subject to two additional constraints on $\chi$ given in [5].

Finally, consider the cases where $n=3$ and $n=4$. The associated Killing spinors both have $1 / 8$ of 16 real degrees of freedom. The solutions are characterised by the addition of one (two) additional off-diagonal entries in $B_{m n}$ for $n=3(n=4)$ [5]. In both cases, the internal metric $G_{m n}$ stays diagonal. It is straightforward to modify these solutions along similar lines as the ones discussed above, and it can be checked that these modified solutions satisfy the Killing spinor equations (3.2).

The modifications are again all encoded in one single holomorphic function $f$. 
Next, we would like to determine the holomorphic function $f$ by demanding that the modified solution should have finite energy [4]. This time, computing the integral (2.12) yields (in units where $8 \pi G_{N}=1$ )

$$
E=i n \int d \omega d \bar{\omega} \frac{\partial_{\omega} f \partial_{\bar{\omega}} \bar{f}}{(f+\bar{f})^{2}}=i n \int d z d \bar{z} \frac{\partial_{z} \hat{f} \partial_{\bar{z}} \overline{\hat{f}}}{(\hat{f}+\overline{\hat{f}})^{2}}
$$

where $\omega=a \ln z$ and where this time $\hat{f}=\frac{n+2}{2 \pi} f$.

As before, by using the j-function map $j(\hat{f})=z$, the energy (5.11) can be made finite and is given by

$$
E=n \frac{\pi}{6}
$$

Note that this is half the amount of energy carried by the solutions with two electric charges that we discussed in section 4 .

By expanding $j(\hat{f})=e^{2 \pi \hat{f}}+744+\mathcal{O}\left(e^{-2 \pi \hat{f}}\right)=z$ we see that $f(\omega)=$ $\frac{1}{n+2}\left(\omega-744 e^{-\omega}+\ldots\right)$, which indeed reproduces the correct asymptotic behaviour at $\omega \rightarrow \infty$. Thus we see that, by demanding the asymptotic behaviour of $f$ to be modified to $f=\frac{2 \pi}{n+2} j^{-1}(z)$, the associated energy becomes finite.

We note that the curvature scalar $\mathcal{R}$ blows up at the special point $\hat{f}=1$.

The solutions discussed above represent one-center solutions. They can again be generalised to multi-center solutions via $j(\hat{f}(z))=P(z) / Q(z)$, where $P(z)$ and $Q(z)$ are polynomials in $z$ with no common factors.

\section{Eleven dimensional interpretation}

Heterotic string theory compactified on a seven-torus is related to M-theory compactified on $S_{1} / Z_{2} \times T_{7}$ [18]. Hence, our solutions should have an eleven dimensional interpretation in terms of configurations of intersecting membranes (M2), 5-branes (M5), M-waves and Kaluza-Klein M-monopoles, which are all supersymmetric solutions to the low-energy effective action of M-theory. These four basic solutions all preserve $1 / 2$ of the elevendimensional supersymmetry. (For a review see [3, 19, 20] and references therein.) In tendimensional heterotic string theory, the basic supersymmetric solutions, when reducing from eleven dimensions, are a fundamental string (coming from M2), a wave, a KK monopole and a NS 5-brane. Each of these ten-dimensional basic solutions preserve $1 / 2$ of 16 supersymmetry. Some of the configurations that one gets when considering various combinations of these objects, when reduced down to three dimensions, should 
correspond to the solutions constructed in [5]. The following interpretation emerges when comparing the ten-dimensional metric of each of these four basic building blocks with the ten-dimensional ansatz for the vielbein (2.4): the wave, when reduced to three dimensions, gives rise to solutions with non-zero $A_{\mu}^{(1) m}$, which are the gauge fields coming from the reduction of $G_{M N}^{(10)}$; the fundamental string gives rise to solutions with nonzero $A_{\mu m}^{(2)}$, coming from the reduction of $B_{M N}^{(10)}$; the NS 5-branes give rise to solutions with non-zero off-diagonal internal $B_{m n}$ components and the Kaluza-Klein monopoles to solutions with non-zero off-diagonal internal metric $G_{m n}$. We will now review the four basic supersymmetric solutions and we will discuss their reduction to three dimensions in detail.

The eleven-dimensional M-branes, M-waves and M-monopoles are solutions to the lowenergy effective action of M-theory, given by $D=11$ supergravity. The bosonic action contains a metric $g_{M N}$ and a three-form potential $A_{M N P}$, with field strength $F_{M N P Q}=$ $24 \nabla_{[M} A_{N P Q]}:$

$$
S=\int \sqrt{-g}\left\{R-\frac{1}{12} F^{2}-\frac{1}{432} \epsilon^{M_{1} \ldots M_{11}} F_{M_{1} \ldots M_{4}} F_{M_{5} \ldots M_{8}} A_{M_{9} \ldots M_{11}}\right\} .
$$

Supersymmetric solutions to the equations of motion of this action can be obtained by looking for backgrounds that admit 32-component Majorana spinors $\varepsilon$ for which the supersymmetry variation of the gravitino field $\psi_{M}$ vanishes.

The M2-brane solution has the form [21]

$$
d s_{11}^{2}=H^{1 / 3}\left[\frac{1}{H}\left(-d t^{2}+d x_{1}^{2}+d x_{11}^{2}\right)+\left(d x_{2}^{2}+\ldots d x_{9}^{2}\right)\right]
$$

with

$$
F_{t 11 \alpha}=\frac{c}{2} \frac{\partial_{\alpha} H}{H^{2}}, \quad H=H\left(x_{2}, \ldots, x_{9}\right), \quad \nabla^{2} H=0, \quad c= \pm 1 .
$$

The solution admits Killing spinors $\varepsilon=H^{-1 / 6} \eta$ with the constant spinor $\eta$ satisfying $\hat{\Gamma}_{0111} \eta=c \eta$, where $\hat{\Gamma}_{0 \ldots p} \equiv \hat{\Gamma}_{0} \ldots \hat{\Gamma}_{p}$ is the product of $p+1$ distinct Gamma matrices in an orthonormal frame. Given that $\left(\hat{\Gamma}_{0111}\right)^{2}=1$ and $\operatorname{Tr} \hat{\Gamma}_{0111}=0$, this solution admits 16-component Killing spinors and preserves half of the supersymmetry.

The single harmonic function determining the solution depends on the orthogonal directions to the 2-brane, $\vec{x}=\left\{x_{2}, \ldots, x_{9}\right\}$. The M2-brane carries electric four-form charge $Q_{e}$ defined as the integral of the seven-form $* F$ around a seven-sphere that surrounds the brane. $c=1$ corresponds to a M2-brane and $c=-1$ to an anti-M2-brane.

We now dimensionally reduce the membrane to ten dimensions to obtain the fundamental string (NS1) by using that 22

$$
d s_{11}^{2}=e^{2 / 3 \Phi^{(10)}} d x_{11}^{2}+e^{-1 / 3 \Phi^{(10)}} d s_{10}^{2} .
$$


Thus, $e^{2 \Phi^{(10)}}=H^{-2}$, and

$$
d s_{10}^{2}=H^{-1}\left(-d t^{2}+d x_{1}^{2}\right)+d x_{2}^{2}+\ldots+d x_{9}^{2} .
$$

Here, $d s_{10}^{2}$ denotes the ten-dimensional line element in the string frame. In order to dimensionally reduce the fundamental string to three dimensions, we take $H$ to be a function of two transverse coordinates only, $H=H\left(x_{8}, x_{9}\right)$, subject to $\nabla^{2} H=0$, where $\nabla^{2}$ now denotes a two-dimensional flat Laplacian. We can take the coordinates $x_{8}$ and $x_{9}$ to be either cartesian or cylindrical. Both coordinate choices, being related by a conformal transformation, are compatible with $\nabla^{2}$ being a flat Laplacian. In the following, we take $x_{8}$ and $x_{9}$ to be cylindrical coordinates $\left(\omega=x_{8}+i x_{9}=\hat{r}+i \hat{\theta}\right)$.

The dimensional reduction of the fundamental string solution to three-dimensions is now obtained by demanding that the line element take the form

$$
d s_{10}^{2}=e^{2 \phi^{(3)}} g_{\mu \nu}^{E(3)} d x^{\mu} d x^{\nu}+G_{m n}^{(7)} d y^{m} d y^{n}
$$

where $G_{m n}^{(7)}$ is the seven-dimensional internal metric with internal coordinates $y^{m}$, and $g_{\mu \nu}^{E(3)}$ is the three-dimensional Einstein metric. Compatibility of the ten-dimensional action and of the three-dimensional action in the Einstein frame requires that $e^{2 \phi^{(3)}}=$ $e^{2 \Phi^{(10)}}\left(\operatorname{det} G_{m n}^{(7)}\right)^{-1}=H^{-1}$ and the three-dimensional space-time line element in the Einstein frame is

$$
d s^{2}=-d t^{2}+H d \omega d \bar{\omega}
$$

In $D=10$, the fundamental string couples to the antisymmetric 2-tensor with $B_{t x_{1}} \propto$ $H^{-1}$, which in $D=3$ yields that $A_{t m}^{(2)}=\frac{c}{2} H^{-1}$, or $F_{t \alpha m}^{(2)}=\frac{c}{2} \frac{\partial_{\alpha} H}{H^{2}}$, where $m=1$.

The solution corresponding to the eleven-dimensional M5-brane is of the form [23]

$$
d s_{11}^{2}=H^{2 / 3}\left[\frac{1}{H}\left(-d t^{2}+d x_{1}^{2}+\ldots d x_{5}^{2}\right)+\left(d x_{6}^{2}+\ldots+d x_{9}^{2}+d x_{11}^{2}\right)\right]
$$

with

$$
F_{\alpha_{1} \ldots \alpha_{4}}=\frac{c}{2} \epsilon_{\alpha_{1} \ldots \alpha_{5}} \partial_{\alpha_{5}} H, \quad H=H\left(x_{6}, \ldots, x_{9}, x_{11}\right), \quad \nabla^{2} H=0, \quad c= \pm 1,
$$

where $\epsilon_{\alpha_{1} \ldots \alpha_{5}}$ is the flat $D=5$ alternating symbol.

The solution admits 16-component Killing spinors $\varepsilon=H^{-1 / 2} \eta$ with $\eta$ satisfying $\hat{\Gamma}_{012345} \eta=c \eta$. The M5-brane carries magnetic four-form charge $Q_{m}$ obtained by integrating $F$ around a four-sphere that surrounds the M5-brane. Here again, $c= \pm 1$ corresponding to a M5- and an anti-M5-brane respectively.

Using (6.4), we find that the M5-brane reduces to a NS 5-brane in ten dimensions, with metric

$$
d s_{10}^{2}=-d t^{2}+d x_{1}^{2}+\ldots+d x_{5}^{2}+H\left(d x_{6}^{2}+\ldots+d x_{9}^{2}\right)
$$


Here $e^{\Phi^{(10)}}=H$. Setting $\omega=x_{8}+i x_{9}$ and taking $H=f(\omega)+\bar{f}(\bar{\omega})$, we find that, by using (6.6), the three-dimensional line element is again given by (6.7), with $e^{\phi^{(3)}}=1$. Reducing $F_{\alpha_{1} \alpha_{2} \alpha_{3} \alpha_{4}}$ down to three dimensions gives rise to $H_{67 \alpha}=\partial_{\alpha} B_{67}$ with $H_{67 \hat{r}} \propto$ $\partial_{\hat{\theta}} H=i \partial_{\hat{r}}(f-\bar{f}), \quad H_{67 \hat{\theta}} \propto-\partial_{\hat{r}} H=i \partial_{\hat{\theta}}(f-\bar{f})$. So it follows that $B_{67} \propto i(f-\bar{f})$ which is in accordance with the expressions for the internal $B_{m n}$ field given in sections 4 and 5 . The wave solution in ten dimensions is given by the metric (with $e^{\phi^{(10)}}=1$ ) [24]

$$
d s_{10}^{2}=-d t^{2}+d y_{1}^{2}+(H-1)\left(d t-d y_{1}\right)^{2}+\left(d x_{2}^{2}+\ldots+d x_{9}^{2}\right),
$$

or, with $y_{1}=t+c x_{1}$,

$$
d s_{10}^{2}=2 c d t d x_{1}+H d x_{1}^{2}+\left(d x_{2}^{2}+\ldots+d x_{9}^{2}\right) .
$$

This corresponds precisely to our $n=1$ solutions carrying one electric charge, with a gauge field $A_{\mu}^{(1) a}=A_{\mu}^{(1) m} e_{m}^{a}$ coming from the reduction of the ten-dimensional metric $G_{M N}^{(10)}$. Indeed, the ten-dimensional line element (2.4) used in the reduction is

$$
d s_{10}^{2}=\left(e^{2 \phi^{(3)}} g_{\mu \nu}^{E(3)}+A_{\mu}^{(1) a} \eta_{a b} A_{\nu}^{(1) b}\right) d x^{\mu} d x^{\nu}+2 A_{\mu}^{(1) a} \eta_{a b} e_{n}^{b} d x^{\mu} d y^{n}+G_{m n}^{(7)} d y^{m} d y^{n} .
$$

Inserting (5.3) and (5.8) into (6.13), we recover (6.12), with $c=\eta_{\alpha_{1}}$, where $x_{1}, x_{2} \ldots x_{7}$ belong to the seven-dimensional torus $T_{7}$ and $\omega=x_{8}+i x_{9}$.

The Kaluza-Klein monopole in ten dimensions is given by the metric [25]

$$
d s_{10}^{2}=-d t^{2}+H d x_{i}^{2}+H^{-1}\left(d z+A_{i} d x_{i}\right)^{2}+d x_{1}^{2}+\ldots+d x_{5}^{2}, \quad i=6,8,9,
$$

with $H=H\left(x_{i}\right), \quad F_{i j}=\partial_{i} A_{j}-\partial_{j} A_{i}=c \varepsilon_{i j k} \partial_{k} H, \quad e^{2 \Phi^{(10)}}=1, \quad c= \pm$.

In five dimensions, the metric reduces to

$$
d s_{5}^{2}=-d t^{2}+H d x_{i}^{2}+H^{-1}\left(d z+A_{i} d x_{i}\right)^{2},
$$

with $e^{2 \phi^{(5)}}=1$.

Now, let $H=H\left(x_{8}, x_{9}\right)$. We can set $A_{8}=A_{9}=0$. Then, $\partial_{8} A_{6}=-c \partial_{9} H, \partial_{9} A_{6}=c \partial_{8} H$. The metric is now

$$
\begin{aligned}
d s_{5}^{2} & =-d t^{2}+H\left(d x_{8}^{2}+d x_{9}^{2}\right)+H d x_{6}^{2}+H^{-1}\left(d z+A_{6} d x_{6}\right)^{2} \\
& =e^{2 \phi^{(3)}} g_{\mu \nu}^{E(3)} d x^{\mu} d x^{\nu}+G_{m n} d y^{m} d y^{n},
\end{aligned}
$$

with $e^{\phi^{(3)}}=1$ and the off-diagonal internal metric is given by

$$
G_{m n}=\left(\begin{array}{cc}
H+A_{6}^{2} H^{-1} & A_{6} H^{-1} \\
A_{6} H^{-1} & H^{-1}
\end{array}\right) .
$$

We now present the supersymmetric heterotic solutions discussed in sections 4 and 5 as dimensionally reduced solutions corresponding to orthogonally intersecting strings, NS 5-branes, waves and KK monopoles in ten dimensions. 


\subsection{Solutions carrying two electric charges}

As discussed in section 4 , the three-dimensional space-time line element in the Einstein frame takes the form

$$
d s^{2}=-d t^{2}+H^{2 n} d \omega d \bar{\omega}
$$

Consider first the case $n=1$. This solution with diagonal and constant internal vielbein $e_{m}^{a}$ and $B_{m n}=0$ corresponds to a fundamental string and a wave in $D=10$, with the wave travelling along the string.

In $D=10$, the solution is

$$
d s_{10}^{2}=\left(H_{s}\right)^{-1}\left[-d t^{2}+d y_{1}^{2}+\left(H_{w}-1\right)\left(d t-d y_{1}\right)^{2}\right]+d x_{2}^{2}+\ldots+d x_{9}^{2}
$$

or, using $(6.12$

$$
d s_{10}^{2}=\left(H_{s}\right)^{-1}\left[2 c d t d x_{1}+H_{w} d x_{1}^{2}\right]+d x_{2}^{2}+\ldots+d x_{9}^{2}
$$

The ten dimensional dilaton is given by $e^{\Phi^{(10)}}=H_{s}^{-1}$. Note that the line element reduces to the wave (6.12) or string (6.5) line elements when we respectively set $H_{s}$ and $H_{w}$ to 1. We now set $H_{s}=H_{w}=H\left(x_{8}, x_{9}\right)$. Comparing (6.20) with $(6.13)$, we find that the internal metric is constant and that consequently $e^{\phi^{(3)}}=H^{-1}$. Furthermore the gauge field is given by $A_{t}^{(1) a}=A_{t}^{(1) m} e_{m}^{a}=c H^{-1}$, where $a=1$. Note that this is in accordance with (4.3) and (4.8).

Since the wave travels along the string, they are both characterized by Killing spinors which obey the same condition $\hat{\Gamma}_{01} \eta=c_{1} \eta$. This solution thus preserves $1 / 2$ of 16 supersymmetry.

Note however that, as described in [5], this purely electric solution can be dualized to a solitonic solution represented by one off-diagonal entry in the internal metric as well as one non-constant entry in the internal $B_{m n}$-matrix. These off-diagonal contributions play the role of 'magnetic' charge from the ten-dimensional point of view and correspond respectively to adding a KK monopole and a NS 5-brane, with a common 5+1-dimensional worldvolume. (This is one of two possible intersection patterns of a KK monopole and a NS 5-brane in $D=10$ [20].) Taking the 5-brane to be oriented along the hyperplane $\{3,4,5,6,7\}$, this configuration is characterized by $\hat{\Gamma}_{034567} \eta=c_{2} \eta$ and $\hat{\Gamma}_{1289} \eta=c_{3} \eta$. The 5 -brane and monopole each break $1 / 2$ of 16 supersymmetry. Since, however, the product of the two Gamma matrix projection operators gives the ten-dimensional chirality operator $\Gamma_{11}$, this pair preserves again 1/2 of 16 supersymmetry. (The ten-dimensional chirality operator, in our notation [5], is $\Gamma_{11}= \pm \gamma^{0} \gamma^{1} \gamma^{2} \gamma^{4} \otimes i \mathbf{I}_{8}$. $\Gamma_{11} \varepsilon=\varepsilon$ is then equivalent to conditions (3.7)). The ten-dimensional line element of this configuration is given 
by

$$
d s_{10}^{2}=-d t^{2}+d x_{3}^{2}+\ldots+d x_{7}^{2}+H_{5} H_{K K}^{-1}\left(\beta d z+A_{2} d x_{2}\right)^{2}+H_{5} H_{K K}\left(d x_{2}^{2}+d x_{8}^{2}+d x_{9}^{2}\right) .
$$

Note that this line element reduces to (6.10) and (6.14) (up to a relabelling of the coordinates) when we respectively set $H_{K K}$ and $H_{5}$ to 1 . The ten-dimensional dilaton is given by $e^{\Phi^{(10)}}=H_{5}$. Setting $H_{5}=H_{K K}=H\left(x_{8}, x_{9}\right)$, and comparing with (6.6), yields a constant three-dimensional dilaton as well as the three-dimensional line element (6.18) with $n=1$. Furthermore, the non-constant part of the internal metric $G_{m n}$ is found here to be

$$
G_{m n}=\left(\begin{array}{cc}
\beta^{2} & \beta A_{2} \\
\beta A_{2} & A_{2}^{2}+H^{2}
\end{array}\right),
$$

where $\beta=\sqrt{G_{11}}$ and where $\partial_{8} A_{2}=-c \partial_{9} H, \partial_{9} A_{2}=c \partial_{8} H$. For $H=f+\bar{f}$, it then follows that $A_{2}=-i c(f-\bar{f})$. This is indeed the off-diagonal internal metric given in [5].

Consider next the solution with $n=2$. There is now, in addition to the two electric charges (corresponding to having a wave and a string), one non-constant off-diagonal entry in the internal metric as well as one non-constant entry in the $B_{m n}$-matrix. In view of the discussion above, this amounts to adding a NS 5-brane and a KK monopole to (6.20). The whole configuration preserves $1 / 4$ of 16 supersymmetry. The ten-dimensional line element thus includes a wave, a fundamental string, a 5-brane and a monopole. The wave is along the string and both are in the worldvolume of the NS 5-brane and KK monopole. The 5-brane and monopole have common worldvolumes. The associated line element is given by

$$
\begin{aligned}
d s_{10}^{2}= & H_{s}^{-1}\left[2 c d t d x_{1}+H_{w} d x_{1}^{2}\right]+d x_{2}^{2}+\ldots+d x_{5}^{2}+H_{5} H_{K K}^{-1}\left(d x_{6}+A_{7} d x_{7}\right)^{2} \\
& +H_{5} H_{K K}\left(d x_{7}^{2}+d x_{8}^{2}+d x_{9}^{2}\right)
\end{aligned}
$$

and the ten-dimensional dilaton is given by $e^{\Phi^{(10)}}=H_{s}^{-1} H_{5}$. We now identify $H_{s}=H_{w}=$ $H_{5}=H_{K K}=H\left(x_{8}, x_{9}\right)$. It is easy now to check that the ten-dimensional line element gives rise to the correct three-dimensional space-time metric (6.18) with $n=2$ as well as to the off-diagonal internal metric given in (4.9). This is done by sending $x_{7} \longrightarrow \frac{x_{7}}{D}$ (where $D=\frac{\sqrt{\left|\alpha_{4} \alpha_{11}\right|}}{\sqrt{\left|\alpha_{2} \alpha_{9}\right|}}$ ) and by relabelling the coordinates.

Consider next the solution with $n=3$. This solution is obtained from the $n=2$ solution by adding an additional off-diagonal entry in both the internal metric $G_{m n}$ and in the $B_{m n}$-matrix. This amounts to adding an additional NS 5-brane and a KK monopole to the line element $(6.23)$ in the following way:

$$
\begin{aligned}
d s_{10}^{2}= & H_{s}^{-1}\left[2 c d t d x_{1}+H_{w} d x_{1}^{2}\right]+d x_{2}^{2}+d x_{3}^{2}+H_{5} H_{K K}^{-1}\left(d x_{4}+A_{5} d x_{5}\right)^{2}+H_{5} H_{K K} d x_{5}^{2} \\
& +H_{5} H_{K K}^{-1}\left(d x_{6}+A_{7} d x_{7}\right)^{2}+H_{5} H_{K K} d x_{7}^{2}+H_{5}^{2} H_{K K}^{2}\left(d x_{8}^{2}+d x_{9}^{2}\right) .
\end{aligned}
$$


Note that the 5-branes intersect orthogonally in a 3-brane 19 and that the KK monopoles have a common three-dimensional worldvolume [20]. The ten-dimensional dilaton is given by $e^{\Phi^{(10)}}=H_{s}^{-1} H_{5}^{2}$. This configuration preserves $1 / 8$ of 16 supersymmetry.

Finally consider our solution with $n=4$. It is obtained by adding an additional offdiagonal entry both in the $G_{m n}$ sector and in the $B_{m n}$ sector. This is achieved by adding yet an additional monopole and an additional 5-brane. The resulting ten-dimensional line element is given by

$$
\begin{aligned}
d s_{10}^{2}= & H_{s}^{-1}\left[2 c d t d x_{1}+H_{w} d x_{1}^{2}\right]+H_{5} H_{K K}^{-1}\left(d x_{2}+A_{3} d x_{3}\right)^{2}+H_{5} H_{K K} d x_{3}^{2} \\
& +H_{5} H_{K K}^{-1}\left(d x_{4}+A_{5} d x_{5}\right)^{2}+H_{5} H_{K K} d x_{5}^{2}+H_{5} H_{K K}^{-1}\left(d x_{6}+A_{7} d x_{7}\right)^{2} \\
& +H_{5} H_{K K} d x_{7}^{2}+H_{5}^{3} H_{K K}^{3}\left(d x_{8}^{2}+d x_{9}^{2}\right) .
\end{aligned}
$$

The ten-dimensional dilaton is given by $e^{\Phi^{(10)}}=H_{s}^{-1} H_{5}^{3}$. Note that adding these two additional objects does not break any further supersymmetry, since the Gamma projection operator for the last 5-brane is given by the product of the Gamma operators of the wave and the two other 5 -branes. Thus, this configuration also preserves $1 / 8$ of 16 supersymmetry.

\subsection{Solutions carrying one electric charge}

In this case, the space-time line element in the Einstein frame has the form

$$
d s^{2}=-d t^{2}+H^{n} d \omega d \bar{\omega} .
$$

The $n=1$ solution corresponds in ten dimensions to a wave and its reduction is the same as in (6.13) and below. The wave preserves $1 / 2$ of 16 supersymmetry.

The $n=2$ case can be described in terms of a wave and one NS 5-brane, since a nonconstant entry in the $B_{m n}$ matrix is added. The ten-dimensional metric describing this pair is given by

$$
d s_{10}^{2}=2 c d t d x_{1}+H_{w} d x_{1}^{2}+d x_{2}^{2}+d x_{3}^{2}+d x_{4}^{2}+d x_{5}^{2}+H_{5}\left(d x_{6}^{2}+\ldots+d x_{9}^{2}\right)
$$

and the ten-dimensional dilaton by $e^{\Phi^{(10)}}=H_{5}$.

This solution preserves $1 / 4$ of 16 supersymmetry. Reducing according to (6.13), we recover our solution given in (5.9) (up to relabelling of the coordinates) with $H_{w}=H_{5}=$ $H\left(x_{8}, x_{9}\right), e^{-2 \phi^{(3)}}=H, A_{t}^{(1) a=1}=c / \sqrt{H}$, and $G_{11}=G_{66}=G_{77}=H$.

The $n=3(n=4)$ case corresponds in the same way to two (three) orthogonally intersecting 5-branes and a wave, where each pair of 5-branes intersects over a 3-brane 
[19]. For instance, the ten-dimensional line element for the $n=4$ case is given by

$d s_{10}^{2}=2 c d t d x_{1}+H_{w} d x_{1}^{2}+H_{5}\left(d x_{2}^{2}+d x_{3}^{2}\right)+H_{5}\left(d x_{4}^{2}+d x_{5}^{2}\right)+H_{5}\left(d x_{6}^{2}+d x_{7}^{2}\right)+H_{5}^{3}\left(d x_{8}^{2}+d x_{9}^{2}\right)$

and the ten-dimensional dilaton by $e^{\Phi^{(10)}}=H^{3}$, where we have again identified the harmonic functions $H_{w}=H_{5}=H\left(x_{8}, x_{9}\right)$.

By using the same argument as in the previous subsection, we conclude that both these cases preserve $1 / 8$ of 16 supersymmetry.

\section{Conclusions}

We showed that the static supersymmetric solutions constructed in [5] can be turned into finite energy solutions, which we computed. The energy of the solutions carrying one electric charge was found to be given by $E=n \frac{\pi}{6}$, whereas the energy of the solutions carrying two electric charges was found to be $E=2 n \frac{\pi}{6}$.

The U-duality group of the low-energy heterotic theory is $O(8,24 ; \mathbb{Z})$ 四. Solutions which are obtained by $O(8,24 ; \mathbb{Z})$ transformations from the ones discussed above will also have finite energy. For instance, the compactified heterotic string solutions of [7, 8] will have the same energies as the solutions carrying one electric charge discussed in section 5, and similarly for the three-dimensional solutions obtained by compactifying a wave and up to three NS 5-branes and Kaluza-Klein monopoles intersecting orthogonally in ten dimensions.

The curvature scalar $\mathcal{R}$ associated with the solutions discussed in sections 4 and 5 is regular everywhere with the exception of the special point $\phi=0$. It would be interesting to understand the physics at this special point in moduli space further.

\section{Acknowledgement}

We would like to thank I. Bakas, K. Behrndt, R. Khuri, D. Lüst, S. Mahapatra, T. Mohaupt and J. Schwarz for valuable discussions. One of us (G. L. C.) is grateful for the hospitality at the Institute of Physics of the Humboldt University Berlin, where part of the work was carried out.

\section{References}

[1] M. J. Duff, R. R. Khuri and J. X. Lu, Phys. Rep. 259 (1995) 213, hep-th/9412184. 
[2] J. G. Russo, hep-th/9703118.

[3] J. P. Gauntlett, hep-th/9705011.

[4] A. Sen, Nucl. Phys. B434 (1995) 179, hep-th/9408083.

[5] I. Bakas, M. Bourdeau and G. L. Cardoso, hep-th/9706032.

[6] N. Marcus and J. Schwarz, Nucl. Phys. B228 (1983) 145.

[7] A. Dabholkar, G. Gibbons, J. Harvey and F. R. Ruiz, Nucl. Phys. B340 (1990) 33;

A. Dabholkar and J. Harvey, Phys. Rev. Lett. 63 (1989) 719.

[8] M. J. Duff, S. Ferrara, R. R. Khuri, J. Rahmfeld, Phys. Lett. B356 (1995) 479, hep-th/9506057.

[9] B. R. Greene, A. Shapere, C. Vafa and S.-T. Yau, Nucl. Phys. B337 (1990) 1.

[10] G. W. Gibbons, M. B. Green and M. J. Perry, Phys. Lett. B370 (1996) 37, hepth/9511080.

[11] S. Ferrara, C. Kounnas and M. Porrati, Phys. Lett. B181 (1986) 263;

M. Terentev, Sov. J. Nucl. Phys. 49 (1989) 713.

[12] J. Maharana and J. H. Schwarz, Nucl. Phys. B390 (1993) 3, hep-th/9207016;

S. Hassan and A. Sen, Nucl. Phys. B375 (1992) 103, hep-th/9109038.

[13] E. Cremmer and B. Julia, Nucl. Phys. B159 (1979) 141.

[14] A. Strominger, Nucl. Phys. B343 (1990) 167; Nucl. Phys. B274 (1986) 253.

[15] P. Candelas, G. Horowitz, A. Strominger and E. Witten, Nucl. Phys. B258 (1985) 46.

[16] J. A. Harvey and J. Liu, Phys. Lett. B268 (1991) 40.

[17] A. Peet, Nucl. Phys. B456 (1995) 732, hep-th/9506200.

[18] P. Hořava and E. Witten, Nucl. Phys. B460 (1996) 506, hep-th/9510209.

[19] A. A. Tseytlin, Nucl. Phys. B475 (1996) 149, hep-th/9604035.

[20] E. Bergshoeff, M. de Roo, E. Eyras, B. Janssen and J. P. van der Schaar, hepth/9704120.

[21] M. J. Duff and K. S. Stelle, Phys. Lett. B253 (1991) 113. 
[22] E. Witten, Nucl. Phys. B443 (1995) 85, hep-th/9503124.

[23] R. Güven, Phys. Lett. B276 (1991) 49.

[24] H. W. Brinkmann, Proc. Nat. Acad. Sci. 9 (1923) 1.

[25] R. D. Sorkin, Phys. Rev. Lett. 51 (1983) 87; D. J. Gross and M. J. Perry, Nucl. Phys. B226 (1983) 29. 